\title{
Postpartum Depressive Symptoms of Mothers with Reciprocal Social Behavior Problems Linked to Increased Aggression in Infants: Hamamatsu Birth Cohort for Mothers and Children (HBC Study)
}

\author{
Ryosuke Asano ${ }^{1,2}$, Kenji J. Tsuchiya ${ }^{1,2 *}$, Nori Takei ${ }^{1,2,3}$, Norio Mori ${ }^{1,2,4}$, HBC Study Team \\ ${ }^{1}$ Research Center for Child Mental Development, Hamamatsu University School of Medicine, Hamamatsu, Japan \\ ${ }^{2}$ Department of Child Development, United Graduate School of Child Development, Osaka University, \\ Kanazawa University, Hamamatsu University School of Medicine, Chiba University and University of Fukui, \\ Hamamatsu, Japan \\ ${ }^{3}$ Division of Psychological Medicine, Institute of Psychiatry, King's College London, London, UK \\ ${ }^{4}$ Department of Psychiatry, Hamamatsu University School of Medicine, Hamamatsu, Japan \\ Email: "tsuchiya@hama-med.ac.jp
}

Received 12 March 2016; accepted 18 April 2016; published 21 April 2016

Copyright (C) 2016 by authors and Scientific Research Publishing Inc.

This work is licensed under the Creative Commons Attribution International License (CC BY).

http://creativecommons.org/licenses/by/4.0/

c) (i) Open Access

\section{Abstract}

Previous studies have led to the assumption that maternal problems in reciprocal social behavior might play an important role in infantile aggression. This study investigated whether maternal problems in reciprocal social behavior were predictive of higher infantile aggression at 18 months of age, and whether maternal postpartum depressive symptoms mediated the association. Participants were 769 mother-child dyads enrolled in the Hamamatsu Birth Cohort for Mothers and Children, in Hamamatsu, Japan. Maternal problems in reciprocal social behavior were evaluated during mid-pregnancy; maternal postpartum depressive symptoms were assessed at 4 weeks after childbirth; and infantile aggression was evaluated at 18 months of age. The results of mediation analysis using structural equation modeling showed that maternal problems in reciprocal social behavior directly increased infantile aggression (estimate $=0.100,95 \% \mathrm{CI}[0.011,0.186]$ ), and indirectly increased infantile aggression via maternal postpartum depressive symptoms (estimate $=0.027,95 \%$ CI $[0.010,0.054])$, even after controlling for covariates. This is the first study to demonstrate two separate processes in the effects of maternal problems in reciprocal social behavior on early infantile aggression.

${ }^{*}$ Corresponding author.

How to cite this paper: Asano, R., Tsuchiya, K. J., Takei, N., \& Mori, N., HBC Study Team (2016). Postpartum Depressive Symptoms of Mothers with Reciprocal Social Behavior Problems Linked to Increased Aggression in Infants: Hamamatsu Birth Cohort for Mothers and Children (HBC Study). Psychology, 7, 546-556. http://dx.doi.org/10.4236/psych.2016.74056 


\section{Keywords}

\section{Aggression, Infancy, Reciprocal Social Behavior, Postpartum Depressive Symptoms, Birth Cohort}

\section{Introduction}

A growing body of literature indicated that aggression can be observed during early infancy, in particular during the first year after birth, with a typical peak of aggression between the second and fourth years of life. These findings are consistent with infantile aggression assessments using parental reports (Alink et al., 2006; Hay et al., 2014; Tremblay et al., 2004) and a direct observation of peer interactions (Hay, Nash et al., 2011). Aggression between approximately six months and three years after birth can be characterized by the use of physical force and expressions of anger directed at family members and peers (Hay, Perra et al., 2010). Furthermore, previous reviews have suggested that early infantile aggression is associated with hostile social cognitive biases and impaired self-regulation of behavior and emotions, which in turn potentially increase antisocial behavior during childhood or later in life (Shonkoff, Boyce, \& McEwen, 2009; Tremblay, 2010). However, to date, little is known about the risk factors associated with early infantile aggression.

It has been theorized that maternal, rather than paternal, factors exert greater influence on the emergence of infantile behaviors including aggression (Bowlby, 1988; Tremblay, 2010). Among these maternal factors, studies have reported that postpartum depressive symptoms (PDS) might increase the risk of their progeny displaying aggression from childhood through adolescence (Barker, Copeland, Maughan, Jaffee, \& Uher, 2012; Barker, Jaffee, Uher, \& Maughan, 2011; Hay, Pawlby, Angold, Harold, \& Sharp, 2003; O’Hara \& McCabe, 2013). Potential explanations for the association between maternal PDS and childhood aggression include poor parenting styles (e.g., unsatisfactory breastfeeding and undesirable sleep practices) and maternal attachment insecurity for their infants (Field, 2010; Jones, Cassidy, \& Shaver, 2015; Murray \& Cooper, 1997). Therefore, it is highly possible that the children of mothers with PDS at four weeks after delivery display higher levels of aggression during early infancy.

Accumulating evidence suggests that individuals with behavioral characteristics that are similar to the autism spectrum disorder are more likely to develop depressive symptoms than those lacking such characteristics (Gerdts \& Bernier, 2011; Ingersoll \& Hambrick, 2011; Sucksmith, Roth, \& Hoekstra, 2011). One study also reported that mothers with deficient in reciprocal social behavior, as indicated by higher scores on the Broader Phenotype Autism Symptoms Scale (BPASS) (Dawson et al., 2007), were associated with an increase of PDS (Asano et al., 2014). Constantino et al. (Constantino, Przybeck, Friesen, \& Todd, 2000; Constantino \& Todd, 2000) have defined problems in reciprocal social behavior as a lack of capacity to engage in emotionally appropriate, mutual, social interaction with others. This definition suggests that the BPASS could be regarded as a valid measure for assessing problems in reciprocal social behavior. Problems in reciprocal social behavior are widely distributed throughout the general population, with a moderate to high link with inheritance (Constantino \& Todd, 2003). Moreover, problems with reciprocal social behavior are known to be moderately correlated with various behavioral problems, such as anxiety, depressive symptoms, and delinquent and aggressive behavior (Constantino et al., 2000). Therefore, we decided to investigate whether mothers with problems in reciprocal social behavior tended to have children that showed higher levels of aggression during early infancy.

Two lines of evidence have led to the assumption that maternal problems in reciprocal social behavior might play an important role in infantile aggression, possibly as a result of the influence of maternal PDS. Maternal problems in reciprocal social behavior might be directly associated with an increase of aggression in children. Maternal problems in reciprocal social behavior could also create a risk factor per se for children developing problems in reciprocal social behavior and subsequently be related to aggressive acts and behavioral problems (Constantino et al., 2000; Constantino \& Todd, 2003). However, to our best knowledge, there are no reported studies that have addressed these issues to date. This study was designed to investigate whether maternal problems in reciprocal social behavior were predictive of higher levels of early infantile aggression and if this association could be explained by maternal PDS.

Using data from a Japanese birth cohort, the current study tested whether maternal problems in reciprocal social behavior were associated with increased infantile aggression at 18 months of age, and whether maternal 
PDS mediated the association between maternal problems in reciprocal social behavior and infantile aggression.

\section{Methods}

\subsection{Procedure}

This study was conducted as a part of an ongoing cohort study in Hamamatsu city in the mainland of Japan, known as the "Hamamatsu Birth Cohort for Mothers and Children (HBC Study)" (Takagai et al., in press; Tsuchiya et al., 2010). Prior to the commencement of the HBC Study, the participating women were given a complete description of the study protocol, and all of them provided written informed consent for her own participation and her infant participation as the infant's legal surrogate. The face-to-face interviews and direct assessments took place in examination rooms situated at our two research sites, including the Hamamatsu University Hospital and Kato Maternity Clinic, during the second trimester of pregnancy, and from 1 to 96 months after delivery. We had previously established that participating women were representative of Japanese mothers in terms of age, socioeconomic status, history of major depressive disorders, and parity, and that the children were representative of Japanese children in birth weight and gestational age (Takagai et al., in press; Tsuchiya et al., 2010).

Hamamatsu University School of Medicine and the University Hospital Ethics Committee approved the protocol of the HBC Study (No. 20-82, 21-114, 22-29, 24-67, 24-237, 25-143, 25-283, E14-062).

\subsection{Participants}

We recruited pregnant women that were expecting to give birth in the two research sites who gave birth between December 2007 and March 2011 ( $N=1202)$. Fifty-four mothers that enrolled in the study more than once because of subsequent births, and 13 mothers that delivered twins were excluded from the following analyses in order to avoid violations of the assumption of independence of each infant. In addition, 423 children were not measured infantile aggression at 18 months of age due to poor health, residential move, or death. Therefore, 769 mothers and their 18-month-old infants were included in the analyses.

\subsection{Measures}

Maternal problems in reciprocal social behavior were assessed during mid-pregnancy using the BPASS (Dawson et al., 2007), which is a measure of behavioral and cognitive characteristics comprising 11 items. The BPASS assesses four domains: the social motivation domain (assessed by two items; e.g., "How comfortable or uncomfortable are you in social situations?”); the expressiveness domain (assessed by four items; e.g., “Observe participant's eye contact both when listening and speaking or playing with examiner”); the conversational skills domain (assessed by two items; e.g., "Observe whether and how frequently the participant offers/asks for excessive detail in conversation”); and the flexibility/range of interests domain (assessed by three items; e.g., "Do you like to have a certain amount of predictability and routine day by day?”). The BPASS has been translated into Japanese and validated (Asano et al., 2014). Trained interviewers administered the face-to-face interviews to the participating women and rated the degree to which each statement applied to each woman (using scales between 1 - 5, 1 - 4, or 1 - 3 depending on the item). Ratings were summed to yield a total score that reflected problems in reciprocal social behavior of each mother. Higher scores represent more problems in reciprocal social behavior.

Maternal PDS was assessed four weeks after childbirth using the Edinburgh Postnatal Depression Scale (EPDS) (Cox \& Holden, 2003), which is a well-validated measure of depressive symptoms comprising 10 items. Mothers were asked to complete the self-report questionnaires in their homes, and return it to our research center by post. Examples of items in the EPDS include "I have blamed myself unnecessarily when things went wrong," "I have felt scared or panicky for no very good reason,” and "I have been so unhappy that I have been crying.” The participating mothers reported the degree to which each statement applied to them using a scale between 0 - 3 . Responses were summed to calculate the PDS scores of each mother. Higher scores represent higher levels of PDS.

Infantile aggression was assessed at 18 months of age using the Cardiff Infant Contentiousness Scale (CICS) (Hay, Perra et al., 2010), which is a measure of the use of physical force and expressions of anger toward family members and peers during infancy comprising 4 items. The CICS included four items: "hits out," "bites," "has angry moods," and "has temper tantrums." Trained interviewers administered the face-to-face interviews to the participating mothers and rated the degree to which each statement applied to the infants using a scale between 0 
= absent and 2 = definitely present. Ratings were summed to yield the infantile aggression scores of each 18-month-old infant. Higher scores represent higher levels of infantile aggression.

Previous studies have indicated that the following covariates, had been assessed in the HBC Study, are associated with early infantile aggression (Alink et al., 2006; Hay, Mundy et al., 2011; Tremblay et al., 2004): (a) maternal age, (b) paternal age, (c) maternal years of education, (d) paternal years of education, (e) marital status of the mother during early pregnancy, (f) annual household income, (g) maternal history of depression and/or anxiety disorders, and (h) infant gender. As with past research (Asano et al., 2014; Matsumoto et al., 2011; Mori et al., 2011), trained interviewers confirmed the history of depression and/or anxiety disorders of mothers using the Structured Clinical Interview for DSM-IV Axis I Disorders (First, Spitzer, Gibbon, \& Williams, 1997). We assessed all covariates during the second trimester of the index pregnancy, with the exception of the infant gender. Demographic characteristics are presented in Table 1.

\subsection{Data Analysis}

First, we calculated descriptive statistics related to maternal problems in reciprocal social behavior, maternal PDS, and infantile aggression. We also examined correlations among the assessed variables. Stata 14.0 (StataCorp, 2015) and R 3.0.2 (R Core Team, 2014) were used for these preliminary analyses.

Next, we performed mediation analysis using structural equation modeling (SEM) with the maximum likelihood estimation to test our hypothetical model which stated that maternal problems in reciprocal social behavior were directly, and indirectly via maternal PDS, were associated with infantile aggression at 18 months of age (Figure 1), even after controlling for covariates. Mediation analysis is a statistical method to test underlying mechanisms through which a predictor affects an outcome by estimating direct and indirect effects (Cheong \& MacKinnon, 2012; Hayes, 2013). The direct effect represents the effect of the predictor on the outcome, whereas the indirect effect represents the effect of the predictor on the outcome via mediators. We first examined whether maternal problems in the reciprocal social behavior influenced infantile aggression at 18 months of age, irrespective of maternal PDS (i.e., total effect; see top of Figure 1). Then, we examined whether maternal problems in reciprocal social behavior directly or indirectly influenced infantile aggression at 18 months of age by including maternal PDS into the model (see bottom of Figure 1). The significance of indirect effect was tested using 95\% bias-corrected bootstrap confidence interval (CI) with the number of resampling set to 1000 (Preacher \& Hayes, 2008). Mplus 7.3 (Muthén \& Muthén, 1998-2012) was used for the mediation analysis.

In addition, we performed a supplemental analysis to rule out the possibility that paternal problems in reciprocal social behavior, which were assessed during mid-pregnancy, simultaneously with maternal problems in reciprocal social behavior, increased the risk for infantile aggression at 18 months of age.

\section{Results}

\subsection{Preliminary Analyses}

Table 2 shows descriptive statistics for the three major variables: maternal problems in reciprocal social behavior during mid-pregnancy, maternal PDS at four weeks after childbirth, and infantile aggression at 18 months

\begin{tabular}{ccc} 
Table 1. Demographic characteristics of participants $(N=769)$. \\
\hline Maternal age at birth (mean) & & \\
Paternal age at birth (mean) & & $31.2 \pm 5.01$ \\
Maternal years of education (mean) & $13.8 \pm 1.92$ \\
Paternal years of education (mean) & $14.1 \pm 2.65$ \\
Marital status at early pregnancy & Married & $747(97.1 \%)$ \\
\hline & Unmarried & $22(2.9 \%)$ \\
Annual household income & $\geq 8$ million yen & $151(19.6 \%)$ \\
& $3-7.9$ million yen & $566(73.6 \%)$ \\
& $<3$ million yen & $52(6.8 \%)$ \\
\hline \multirow{2}{*}{ Maternal history of depression and/or anxiety disorder } & No & $679(88.3 \%)$ \\
& Yes & $90(11.7 \%)$ \\
\hline \multirow{2}{*}{ Infant gender } & Female & $383(49.8 \%)$ \\
& Male & $386(50.2 \%)$ \\
\hline
\end{tabular}


of age. Because means and standard deviations of maternal PDS and infantile aggression showed floor effects, we considered these variables as non-normal distribution in the following analyses.

Table 3 represents the correlations among the assessed variables. We calculated Spearman's rank-order correlations due to floor effects in maternal PDS and infantile aggression. As seen in Table 3, maternal problems in reciprocal social behavior were positively correlated with maternal PDS ( $\rho=0.119, p=0.002)$ and infantile aggression $(\rho=0.078, p=0.020$ ), indicating that mothers with higher scores for problems in reciprocal social

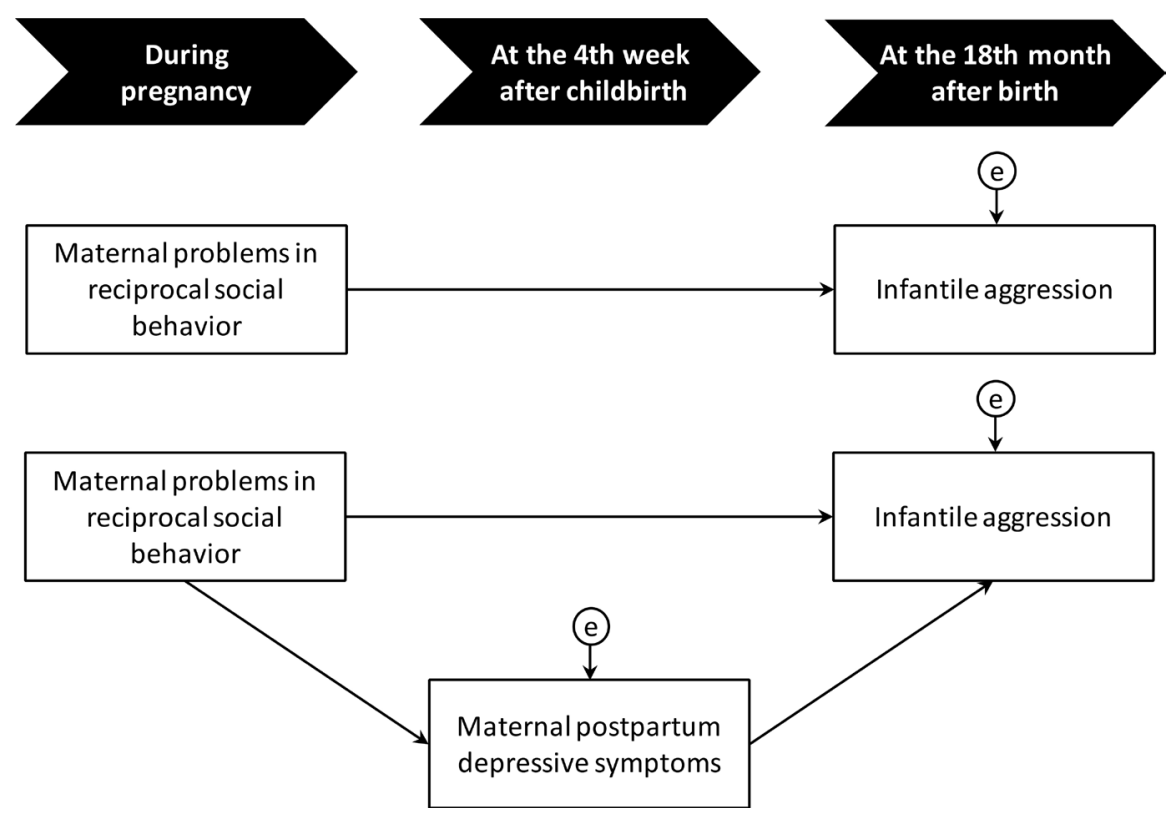

Figure 1. Mediation analysis of maternal postpartum depressive symptoms associated with maternal problems in reciprocal social behavior and infantile aggression.

Table 2. Descriptive statistics of the three major variables.

\begin{tabular}{cccccccc}
\hline & Mean & SD & Range & Skewness & Kurtosis & $\square \omega$ & $N$ \\
\hline Maternal problems in reciprocal social behavior & 13.25 & 1.84 & $11-23$ & 1.14 & 5.03 & 0.54 & 769 \\
Maternal postpartum depressive symptoms & 3.13 & 3.57 & $0-21$ & 1.99 & 7.87 & 0.90 & 710 \\
Infanile aggression & 1.88 & 1.79 & $0-8$ & 0.96 & 3.42 & 0.65 & 769 \\
\hline
\end{tabular}

Note: $S D$ = standard deviation. Problems in reciprocal social behavior were assessed using scores on the Broader Phenotype Autism Symptoms Scale. McDonald's $\omega$ represents internal consistency reliability.

Table 3. Correlations among predictors and maternal postpartum depressive symptoms and infantile aggression $(N=710$ 769).

\begin{tabular}{ccc}
\hline & Maternal postpartum depressive symptoms & Infantile aggression \\
\hline Maternal problems in reciprocal social behavior & $\rho=0.119, p=0.002$ & $\rho=0.078, p=0.030$ \\
Maternal age & $\rho=-0.006, p=0.865$ & $\rho=-0.054, p=0.137$ \\
Paternal age & $\rho=0.000, p=0.991$ & $\rho=-0.067, p=0.062$ \\
Maternal years of education & $\rho=0.029, p=0.436$ & $\rho=-0.109, p=0.003$ \\
Paternal years of education & $\rho=0.012, p=0.753$ & $\rho=-0.107, p=0.003$ \\
Marital status & $\rho=0.003, p=0.935$ & $\rho=0.113, p=0.002$ \\
Annual household income & $\rho=0.042, p=0.262$ & $\rho=0.021, p=0.571$ \\
Maternal history of depression and/or anxiety disorders & $\rho=0.133, p<0.001$ & $\rho=0.113, p=0.002$ \\
\hline Infant gender & $\rho=0.018, p=0.630$ &
\end{tabular}

Note: Problems in reciprocal social behavior were assessed using scores on the Broader Phenotype Autism Symptoms Scale. 
behavior reported higher levels of PDS and had children with higher levels of infantile aggression. In addition, maternal PDS was positively correlated with infantile aggression $(\rho=0.099, p=0.009)$, suggesting that mothers that were higher PDS had children with higher levels of infantile aggression.

\subsection{Maternal Problems in Reciprocal Social Behavior, Maternal PDS, and Infantile Aggression}

As shown in the top of Figure 1, we developed the SEM to investigate the effect of maternal problems in reciprocal social behavior on infantile aggression at 18 months of age. Because maternal PDS and infantile aggression showed non-normality, we applied a censored normal model that does not require the normality assumption and yields robust estimates (Nagin \& Tremblay, 1999; Wang \& Zhang, 2011), although model fit indices (e.g., the comparative fit index and root mean square error of approximation) were not calculated.

The results demonstrated that maternal problems in reciprocal social behavior was positively associated with infantile aggression (estimate $=0.123,95 \%$ CI $[0.032,0.215]$ ), after controlling for covariates including maternal age, paternal age, maternal years of education, paternal years of education, marital status, annual household income, maternal history of depression and/or anxiety disorders, and infant gender (Table 4). Therefore, it was suggested that mothers with problems in reciprocal social behavior had 18-month-old infants with higher levels of aggression.

Next, we conducted a mediation analysis using the SEM to test whether the association between maternal problems in reciprocal social behavior and infantile aggression at 18 months of age could be explained by maternal PDS (the bottom of Figure 1). The results indicated that (a) maternal PDS was positively associated with infantile aggression, and (b) maternal problems in reciprocal social behavior were positively associated with maternal PDS, even after controlling for all covariates (Table 5). SEM indicated that maternal PDS mediated the association between maternal problems in reciprocal social behavior and infantile aggression because 95\% bias-corrected bootstrap CI for the product of unstandardized coefficients (a) and (b) was greater than zero (estimate $=0.027,95 \%$ CI $[0.010,0.054])$. In addition, the direct association between maternal problems in reciprocal social behavior and infantile aggression remained statistically significant (estimate $=0.100,95 \%$ CI $[0.011,0.186])$. In summary, as expected, maternal problems in reciprocal social behavior increased aggression in 18-month-old infants, with maternal PDS partially accounting for the association.

\subsection{Supplemental Analysis}

We re-ran mediation analysis using the SEM to examine whether paternal problems in reciprocal social behavior were associated with aggression at 18 months of age via maternal PDS. The results demonstrated that paternal

Table 4. Structural equation model predicting infantile aggression stemming from maternal problems in reciprocal social behavior $(N=769)$.

\begin{tabular}{|c|c|c|c|}
\hline & Estimate & $95 \% \mathrm{CI}$ & $p$ \\
\hline \multicolumn{4}{|l|}{ Infantile aggression } \\
\hline Maternal problems in reciprocal social behavior & 0.123 & {$[0.032,0.215]$} & 0.008 \\
\hline Maternal age at birth & 0.010 & {$[-0.043,0.062]$} & 0.719 \\
\hline Paternal age at birth & -0.023 & {$[-0.068,0.022]$} & 0.319 \\
\hline Maternal years of education & -0.032 & {$[-0.136,0.071]$} & 0.542 \\
\hline Paternal years of education & -0.065 & {$[-0.139,0.010]$} & 0.091 \\
\hline Marital status at pregnancy (unmarried) & 1.028 & {$[0.010,2.046]$} & 0.048 \\
\hline \multicolumn{4}{|l|}{ Annual household income } \\
\hline 3 - 7.9 million yen & 0.268 & {$[-0.193,0.728]$} & 0.255 \\
\hline$<3$ million yen & 0.451 & {$[-0.336,1.239]$} & 0.261 \\
\hline Maternal history of depression and/or anxiety disorders (yes) & 0.049 & {$[-0.478,0.576]$} & 0.857 \\
\hline Infant gender (male) & 0.103 & {$[-0.235,0.440]$} & 0.551 \\
\hline
\end{tabular}

Note: The reference value for annual household income is $\geq 8$ million yen. Problems in reciprocal social behavior were assessed using scores on the Broader Phenotype Autism Symptoms Scale. 
Table 5. Structural equation model predicting infantile aggression stemming from maternal postpartum depressive symptoms and problems in reciprocal social behavior $(N=769)$.

\begin{tabular}{|c|c|c|c|}
\hline & Estimate & $95 \%$ CI & $p$ \\
\hline \multicolumn{4}{|l|}{ Infantile aggression } \\
\hline Maternal postpartum depressive symptoms & 0.072 & {$[0.029,0.117]$} & 0.001 \\
\hline Maternal problems in reciprocal social behavior & 0.100 & {$[0.011,0.186]$} & 0.027 \\
\hline Maternal age at birth & 0.009 & {$[-0.044,0.061]$} & 0.726 \\
\hline Paternal age at birth & -0.023 & {$[-0.064,0.024]$} & 0.309 \\
\hline Maternal years of education & -0.041 & {$[-0.140,0.059]$} & 0.413 \\
\hline Paternal years of education & -0.062 & {$[-0.145,0.020]$} & 0.142 \\
\hline Marital status at pregnancy (unmarried) & 0.987 & {$[0.043,1.809]$} & 0.022 \\
\hline \multicolumn{4}{|l|}{ Annual household income } \\
\hline 3 - 7.9 million yen & 0.191 & {$[-0.275,0.644]$} & 0.430 \\
\hline$<3$ million yen & 0.480 & {$[-0.313,1.280]$} & 0.250 \\
\hline Maternal history of depression and/or anxiety disorders (yes) & -0.083 & {$[-0.628,0.399]$} & 0.759 \\
\hline Infant gender (male) & 0.076 & {$[-0.260,0.400]$} & 0.659 \\
\hline \multicolumn{4}{|l|}{ Maternal postpartum depressive symptoms } \\
\hline Maternal problems in reciprocal social behavior & 0.380 & {$[0.221,0.576]$} & $<0.001$ \\
\hline Maternal age at birth & -0.013 & {$[-0.107,0.093]$} & 0.794 \\
\hline Paternal age at birth & 0.010 & {$[-0.079,0.088]$} & 0.817 \\
\hline Maternal years of education & 0.133 & {$[-0.042,0.322]$} & 0.159 \\
\hline Paternal years of education & -0.041 & {$[-0.169,0.084]$} & 0.526 \\
\hline Marital status at pregnancy (unmarried) & 0.656 & {$[-1.458,3.754]$} & 0.608 \\
\hline \multicolumn{4}{|l|}{ Annual household income } \\
\hline 3 - 7.9 million yen & 1.265 & {$[0.527,2.107]$} & 0.255 \\
\hline$<3$ million yen & -0.649 & {$[-2.317,0.527]$} & 0.261 \\
\hline Maternal history of depression and/or anxiety disorders (yes) & 2.041 & {$[0.802,3.189]$} & 0.001 \\
\hline Infant gender (male) & 0.287 & {$[-0.303,0.919]$} & 0.358 \\
\hline
\end{tabular}

Note: Reference of annual household income is $\geq 8$ million yen. Problems in reciprocal social behavior were assessed using scores on the Broader Phenotype Autism Symptoms Scale.

problems in reciprocal social behavior were neither directly (estimate $=-0.030,95 \% \mathrm{CI}[-0.110,0.041])$ nor indirectly associated with infantile aggression through the mediation of the maternal PDS (estimate $=0.010,95 \%$ CI [-0.001, 0.028]; table not shown). Therefore, fathers with problems in reciprocal social behavior did not have 18-month-old infants with higher levels of aggression.

\section{Discussion}

This study investigated the influence of maternal problems in reciprocal social behavior and PDS on infantile aggression at 18 months of age using a birth cohort in Japan. We focused on whether maternal problems in reciprocal social behavior increase the likelihood of infantile aggression and whether the association was mediated by maternal PDS after controlling for potential covariates. Our analysis supported both hypotheses and suggested that there are two separate processes influencing the development of early infantile aggression. The first is a direct pathway leading from maternal problems in reciprocal social behavior to infantile aggression; the other is an indirect pathway stemming from maternal problems in reciprocal social behavior to infantile aggression via maternal PDS.

We found a direct pathway between maternal problems in reciprocal social behavior and infantile aggression at 18 months of age. Maternal characteristics are transmitted between generations during the prenatal development of an infant's brain, which is known to result in failures in emotion and behavior regulation, including ag- 
gression (Tremblay, 2010). These characteristics include problems in reciprocal social behavior, such as lower degree of social motivation and communication skills (Constantino et al., 2000; Matson \& Adams, 2014). To our knowledge, this is the first investigation showing that maternal problems in reciprocal social behavior can predict the risk of developing aggression during early infancy.

In addition, we identified an indirect pathway between maternal problems in reciprocal social behavior and infantile aggression at 18 months of age via maternal PDS. The results supported past studies reporting that mothers exhibiting problems in reciprocal social behavior have higher levels of PDS after childbirth (Asano et al., 2014) as well as studies suggesting that mothers with PDS tend to engage in poor parenting styles (Field, 2010). However, maternal mediators other than PDS, including maternal prepartum depressive symptoms, could also explain the direct pathway that we observed. Past studies have reported that maternal prepartum depressive symptoms are a consistent predictor of their children exhibiting aggression and other behavioral problems (Hay, Mundy et al., 2011; Hay, Pawlby, Waters, Perra, \& Sharp, 2010), even after controlling for maternal PDS (Waters, Hay, Simmonds, \& van Goozen, 2014). We did not assess prepartum depressive symptoms using the EPDS, and thus this possibility cannot be ruled out by this study. However, our study design controlled for a maternal history of depression and/or anxiety disorders using a structured interview based on DSM criteria which are more stringent in detecting depressive symptoms than self-report scales such as the EPDS. Therefore, we can assume that an indirect pathway via maternal depressive symptoms during pregnancy did not affect the direct pathway. Another possible mediator of the direct effect is problems in reciprocal social behavior of the infant. Some studies have indicated that problems in reciprocal social behavior are associated with aggression (Constantino et al., 2000) and can be transmitted from parents to offspring (Constantino \& Todd, 2000, 2003). Thus, infants of mothers having problems in reciprocal social behavior may be likely to show more prominent problems in reciprocal social behavior, which in turn causes aggression towards others. These factors are important issues to be addressed in future studies.

There are a number of risk factors that are predictive of aggression among early infants, such as lower parental age, unmarried status, lower income, and infant male gender (Hay, Mundy et al., 2011; Tremblay et al., 2004). Interestingly, however, inclusion or exclusion of these known risk factors did not change the key findings that we reported, because the predictive values of the direct and indirect pathways leading from maternal problems in reciprocal social behavior to infantile aggression remained statistically significant even after controlling for such risk factors. Therefore, the results suggest that maternal problems in reciprocal social behavior directly and indirectly increase in infantile aggression, independent of other known risk factors. Nevertheless, great caution is advised when interpreting the results and conducting clinical interventions based on this study because the observed effects were limited.

As has been suggested in the literature, maternal rather than paternal behaviors generally have a greater influence on the development of children's behaviors (Bowlby, 1988; Tremblay, 2010; Tremblay et al., 2004). Consistent with past research, our additional analysis demonstrated that paternal problems in reciprocal social behavior were neither directly nor indirectly associated with infantile aggression. Indeed, there are no reports of an association between early infantile aggression and paternal behavioral or cognitive characteristics.

There are several limitations to the current study that are important to recognize. First, the sample size was modest, and the majority of participants (91\%) were Japanese. Nevertheless, the generalizability of the results might not be a serious concern because of the high consent rate for enrollment $(>80 \%)$ and the low attrition rate 24 months after childbirth in the HBC Study $(<10 \%)$ (Takagai et al., in press). Second, recall bias could not be completely eliminated because a self-report measure was used to assess maternal PDS. However, the EPDS is the most frequently used scale for measuring PDS, and past studies demonstrated the EPDS sensitivity and specificity in evaluating patients for major depressive disorders (Cox \& Holden, 2003), including the Japanese version of the EPDS (Yamashita, Yoshida, Nakano, \& Tashiro, 2000). Third, we did not collect information pertaining to attachment between the participating mothers and their infants, which leaves room for an explanation of mother-child attachment regarding a direct pathway from maternal problems in reciprocal social behavior to early infantile aggression. Fourth, we could not establish causality, namely whether maternal problems in reciprocal social behavior during pregnancy actually caused changes in maternal PDS 4 weeks after childbirth, which in turn caused changes in the infantile aggression at 18 months of age.

Despite these limitations, the results of this study based on a sample of the Japanese general population suggest that there are two pathways leading to the emergence of infantile aggression at 18 months of age. Moreover, the association between maternal problems in reciprocal social behavior and infantile aggression that we re- 
ported is considered to be highly reliable because these variables were assessed during the face-to-face interviews using a longitudinal study design.

\section{Conclusion}

In summary, the current study suggests that maternal problems in reciprocal social behavior assessed during their pregnancy might be a risk factor for infantile aggression at 18 months of age. Moreover, this association was partly accounted for by maternal PDS at four weeks after childbirth.

\section{Acknowledgements}

The authors acknowledge Professor Dale F. Hay for invaluable comments on earlier drafts. The authors thank Dr. Tetsuo Kato of the Kato Maternity Clinic for conducting HBC Study. The authors are also grateful to Drs. N. Kanayama, H. Itoh, K. Sugihara, M. Sugimura, K. Takeuchi, K. Suzuki, Y. Murakami, Y. Koumura, Y. Miyabe, K. Hirai, Y. Nakamura, R. Koizumi, H. Murakami, Y. Kobayashi-Koumura, and K. Muramatsu-Kato, and all the attending obstetricians for enrolling pregnant women to participate in the study. The authors thank the chief midwife, Ms. Kiyomi Hinoki, and all the midwives and staff at the maternity clinic of the Hamamatsu University School of Medicine, for enrolling participants. The HBC Study team includes Ms. Y. Kugizaki, C. Nakayasu, A. Okumura, Y. Suzuki, N. Kodera, E. Higashimoto, A. Nakamura, E. Kawai, Y. Osuka, T. Nishimura, S. Aoyagi, Y. Sagawa, T. Isobe, M. Yamashita, R. Takabayashi, T. Mori, H. Muraki, M. Narumiya, M. Honda, E. Sato, C. Nishizawa, Mr. R. Nakahara, Drs. T. Harada, Y. Kameno, T. Wakuda, D. Kurita, K. Takebayashi, Y. Iwata, K. Wakusawa, K. Yamada, S. Takagai, K. Suzuki, T. Sugiyama, M. Tsujii, A. A. Pillai, T. Ismail, K. Matsumoto, K. Iwata, C. Shimmura, Y. K. Kuroda, Y. Yoshihara, S. Yamamoto, M. Kawai, K. Nakamura, H. Matsuzaki, G. Sugihara, S. Suda, K. Hirano, Y. Endoh, T. Suzuki.

This research was supported by Developmental Education and Research Encouragement Prize from the Center of Developmental Education and Research of Japan (R. A.), by Grants-in-Aid for Scientific Research (C) and the Strategic Research Program for Brain Sciences ("Integrated research on neuropsychiatric disorders") from the Ministry of Education, Culture, Sports, Science and Technology of Japan (K. J. T.), and by Health and Labor Sciences Research Grant from the Ministry of Health Labour and Welfare of Japan (K. J. T.).

\section{Conflict of Interest}

None of the authors have any conflict of interest to declare.

\section{References}

Alink, L. R., Mesman, J., Van Zeijl, J., Stolk, M. N., Juffer, F., Koot, H. M., \& Van IJzendoorn, M. H. (2006). The Early Childhood Aggression Curve: Development of Physical Aggression in 10- to 50-Month-Old Children. Child Development, 77, 954-966. http://dx.doi.org/10.1111/j.1467-8624.2006.00912.x

Asano, R., Tsuchiya, K. J., Takei, N., Harada, T., Kugizaki, Y., Nakahara, R. et al., HBC Study Team (2014). Broader Autism Phenotype as a Risk Factor for Postpartum Depression: Hamamatsu Birth Cohort (HBC) Study. Research in Autism Spectrum Disorders, 8, 1672-1678. http://dx.doi.org/10.1016/j.rasd.2014.08.010

Barker, E. D., Copeland, W., Maughan, B., Jaffee, S. R., \& Uher, R. (2012). Relative Impact of Maternal Depression and Associated Risk Factors on Offspring Psychopathology. British Journal of Psychiatry, 200, 124-129. http://dx.doi.org/10.1192/bjp.bp.111.092346

Barker, E. D., Jaffee, S. R., Uher, R., \& Maughan, B. (2011). The Contribution of Prenatal and Postnatal Maternal Anxiety and Depression to Child Maladjustment. Depression and Anxiety, 28, 696-702. http://dx.doi.org/10.1002/da.20856

Bowlby, J. (1988). A Secure Base. New York: Basic Books.

Cheong, J., \& MacKinnon, D. (2012). Mediation/Indirect Effects in Structural Equation Modeling. In R. H. Hoyle (Ed.), Handbook of Structural Equation Modeling (pp. 417-435). New York: Guilford Press.

Constantino, J. N., Przybeck, T., Friesen, D., \& Todd, R. D. (2000). Reciprocal Social Behavior in Children with and without Pervasive Developmental Disorders. Journal of Developmental and Behavioral Pediatrics, 21, 2-11. http://dx.doi.org/10.1097/00004703-200002000-00002

Constantino, J. N., \& Todd, R. D. (2000). Genetic Structure of Reciprocal Social Behavior. American Journal of Psychiatry, 157, 2043-2045. http://dx.doi.org/10.1176/appi.ajp.157.12.2043 
Constantino, J. N., \& Todd, R. D. (2003). Autistic Traits in the General Population: A Twin Study. Archives of General Psychiatry, 60, 524-530. http://dx.doi.org/10.1001/archpsyc.60.5.524

Cox, J., \& Holden, J. (2003). Perinatal Mental Health: A Guide to the Edinburgh Postnatal Depression Scale (EPDS). London: Royal College of Psychiatrists.

Dawson, G., Estes, A., Munson, J., Schellenberg, G., Bernier, R., \& Abbott, R. (2007). Quantitative Assessment of Autism Symptom-Related Traits in Probands and Parents: Broader Phenotype Autism Symptom Scale. Journal of Autism and Developmental Disorders, 37, 523-536. http://dx.doi.org/10.1007/s10803-006-0182-2

Field, T. (2010). Postpartum Depression Effects on Early Interactions, Parenting, and Safety Practices: A Review. Infant Behavior \& Development, 33, 1-6. http://dx.doi.org/10.1016/j.infbeh.2009.10.005

First, M. B., Spitzer, R. L., Gibbon, M., \& Williams, J. B. (1997). Structured Clinical Interview for DSM-IV Axis I Disorders (Version 2.0). Arlington, VA: American Psychiatric Publishing.

Gerdts, J., \& Bernier, R. (2011). The Broader Autism Phenotype and Its Implications on the Etiology and Treatment of Autism Spectrum Disorders. Autism Research and Treatment, 2011, Article ID: 545901.

http://dx.doi.org/10.1155/2011/545901

Hay, D. F., Mundy, L., Roberts, S., Carta, R., Waters, C. S., Perra, O. et al. (2011). Known Risk Factors for Violence Predict 12-Month-Old Infants’ Aggressiveness with Peers. Psychological Science, 22, 1205-1211. http://dx.doi.org/10.1177/0956797611419303

Hay, D. F., Nash, A., Caplan, M., Swartzentruber, J., Ishikawa, F., \& Vespo, J. E. (2011). The Emergence of Gender Differences in Physical Aggression in the Context of Conflict between Young Peers. British Journal of Developmental Psychology, 29, 158-175. http://dx.doi.org/10.1111/j.2044-835X.2011.02028.x

Hay, D. F., Pawlby, S., Angold, A., Harold, G. T., \& Sharp, D. (2003). Pathways to Violence in the Children of Mothers Who Were Depressed Postpartum. Developmental Psychology, 39, 1083-1094. http://dx.doi.org/10.1037/0012-1649.39.6.1083

Hay, D. F., Pawlby, S., Waters, C. S., Perra, O., \& Sharp, D. (2010). Mothers’ Antenatal Depression and Their Children’s Antisocial Outcomes. Child Development, 81, 149-165. http://dx.doi.org/10.1111/j.1467-8624.2009.01386.x

Hay, D. F., Perra, O., Hudson, K., Waters, C. S., Mundy, L., Phillips, R., Goodyer, I., Harold, G., Thapar, A., \& van Goozen, S. (2010). Identifying Early Signs of Aggression: Psychometric Properties of the Cardiff Infant Contentiousness Scale. Aggressive Behavior, 36, 351-357. http://dx.doi.org/10.1002/ab.20363

Hay, D. F., Waters, C. S., Perra, O., Swift, N., Kairis, V., Phillips, R., \& Thapar, A. (2014). Precursors to Aggression Are Evident by 6 Months of Age. Developmental Science, 17, 471-480. http://dx.doi.org/10.1111/desc.12133

Hayes, A. F. (2013). Introduction to Mediation, Moderation, and Conditional Process Analysis: A Regression Based Approach. New York: Guilford Press.

Ingersoll, B., \& Hambrick, D. Z. (2011). The Relationship between the Broader Autism Phenotype, Child Severity, and Stress and Depression in Parents of Children with Autism Spectrum Disorders. Research in Autism Spectrum Disorders, 5 , 337-344. http://dx.doi.org/10.1016/j.rasd.2010.04.017

Jones, J. D., Cassidy, J., \& Shaver, P. R. (2015). Parents’ Self-Reported Attachment Styles: A Review of Links with Parenting Behaviors, Emotions, and Cognitions. Personality and Social Psychology Review, 19, 44-76. http://dx.doi.org/10.1177/1088868314541858

Matson, J. L., \& Adams, H. L. (2014). Characteristics of Aggression among Persons with Autism Spectrum Disorders. Research in Autism Spectrum Disorders, 8, 1578-1584. http://dx.doi.org/10.1016/j.rasd.2014.08.004

Matsumoto, K., Tsuchiya, K. J., Itoh, H., Kanayama, N., Suda, S., Matsuzaki, H. et al., The HBC Study Team (2011). Age-Specific 3-Month Cumulative Incidence of Postpartum Depression: The Hamamatsu Birth Cohort (HBC) Study. Journal of Affective Disorders, 133, 607-610. http://dx.doi.org/10.1016/j.jad.2011.04.024

Mori, T., Tsuchiya, K. J., Matsumoto, K., Suzuki, K., Mori, N., \& Takei, N., HBC Study Team (2011). Psychosocial Risk Factors for Postpartum Depression and Their Relation to Timing of Onset: The Hamamatsu Birth Cohort (HBC) Study. Journal of Affective Disorders, 135, 341-346. http://dx.doi.org/10.1016/j.jad.2011.07.012

Murray, L., \& Cooper, P. J. (1997). Effects of Postnatal Depression on Infant Development. Archives of Disease in Childhood, 77, 99-101. http://dx.doi.org/10.1136/adc.77.2.99

Muthén, L. K., \& Muthén, B. O. (1998-2012). Mplus User’s Guide: Statistical Analysis with Latent Variables (7th ed.). Los Angeles, CA: Muthén \& Muthén.

Nagin, D., \& Tremblay, R. E. (1999). Trajectories of Boys’ Physical Aggression, Opposition, and Hyperactivity on the Path to Physically Violent and Nonviolent Juvenile Delinquency. Child Development, 70, 1181-1196. http://dx.doi.org/10.1111/1467-8624.00086

O’Hara, M. W., \& McCabe, J. E. (2013). Postpartum Depression: Current Status and Future Directions. Annual Review of 
Clinical Psychology, 9, 379-407. http://dx.doi.org/10.1146/annurev-clinpsy-050212-185612

Preacher, K. J., \& Hayes, A. F. (2008). Asymptotic and Resampling Strategies for Assessing and Comparing Indirect Effects in Multiple Mediator Models. Behavior Research Methods, 40, 879-891. http://dx.doi.org/10.3758/BRM.40.3.879

R Core Team (2014). R: A Language and Environment for Statistical Computing. Vienna: Foundation for Statistical Computing.

Shonkoff, J. P., Boyce, W. T., \& McEwen, B. S. (2009). Neuroscience, Molecular Biology, and the Childhood Roots of Health Disparities: Building a New Framework for Health Promotion and Disease Prevention. Journal of the American Medical Association, 301, 2252-2259. http://dx.doi.org/10.1001/jama.2009.754

StataCorp (2015). Statistical Software: Release 14. College Station, TX: StataCorp LP.

Sucksmith, E., Roth, I., \& Hoekstra, R. (2011). Autistic Traits below the Clinical Threshold: Re-Examining the Broader Autism Phenotype in the 21st Century. Neuropsychology Review, 21, 360-389. http://dx.doi.org/10.1007/s11065-011-9183-9

Takagai, S., Tsuchiya, K. J., Itoh, H., Kanayama, N., Mori, N., \& Takei, N., on behalf of HBC Study Team (in press). Cohort Profile: Hamamatsu Birth Cohort for Mothers and Children (HBC Study). International Journal of Epidemiology. http://dx.doi.org/10.1093/ije/dyv290

Tremblay, R. E. (2010). Developmental Origins of Disruptive Behaviour Problems: The “Original Sin” Hypothesis, Epigenetics and Their Consequences for Prevention. Journal of Child Psychology and Psychiatry, 51, 341-367. http://dx.doi.org/10.1111/j.1469-7610.2010.02211.x

Tremblay, R. E., Nagin, D. S., Seguin, J. R., Zoccolillo, M., Zelazo, P. D., Boivin, M., \& Japel, C. (2004). Physical Aggression during Early Childhood: Trajectories and Predictors. Pediatrics, 114, e43-e50. http://dx.doi.org/10.1542/peds.114.1.e43

Tsuchiya, K. J., Matsumoto, K., Suda, S., Miyachi, T., Itoh, H., Kanayama, N., Hirano, K., Ohzeki, T., Takeia1, N., \& The HBC Study Team (2010). Searching for Very Early Precursors of Autism Spectrum Disorders: The Hamamatsu Birth Cohort for Mothers and Children (HBC). Journal of Developmental Origins of Health and Disease, 1, 158-173. http://dx.doi.org/10.1017/S2040174410000140

Wang, L., \& Zhang, Z. (2011). Estimating and Testing Mediation Effects with Censored Data. Structural Equation Modeling, 18, 18-34. http://dx.doi.org/10.1080/10705511.2011.534324

Waters, C. S., Hay, D. F., Simmonds, J. R., \& van Goozen, S. H. (2014). Antenatal Depression and Children’s Developmental Outcomes: Potential Mechanisms and Treatment Options. European Child and Adolescent Psychiatry, 23, 957-971. http://dx.doi.org/10.1007/s00787-014-0582-3

Yamashita, H., Yoshida, K., Nakano, H., \& Tashiro, N. (2000). Postnatal Depression in Japanese Women: Detecting the Early Onset of Postnatal Depression by Closely Monitoring the Postpartum Mood. Journal of Affective Disorders, 58, 145-154. http://dx.doi.org/10.1016/S0165-0327(99)00108-1 